|| Print ISSN: 2589-7837 || Online ISSN: 2581-3935 ||

International Journal of Medical Science and Diagnosis Research (IJMSDR)

Available Online at www.ijmsdr.com

NLM (National Library of Medicine ID: 101738824)

Original Research Article

Volume 5, Issue 9; September:2021; Page No. 01-06

\title{
Distribution of ANA Pattern and Autoantibody Profile in Various Autoimmune Disorders -A Tertiary Care Centre Experience.
}

\section{Shabnum Hussain ${ }^{1}$, Preethishree Padil ${ }^{1}$, Ibrahim Masoodi ${ }^{2}$, Rouchelle Tellis ${ }^{1}$, Vidya Pai ${ }^{1}$}

${ }^{1}$ Department of Clinical Microbiology, Yenepoya Medical College Mangalore Karnataka -575018

${ }^{2}$ Department of Medicine, Yenepoya Medical College Mangalore Karnataka -575018

Conflicts of Interest: Nil

Corresponding author: Preethishree Padil. (E-mail: 06.preethi@gmail.com)

DOI: https://doi.org/10.32553/ijmsdr.v5i9.844

\section{Abstract:}

Background: Type of antibodies produced by an individual in response to any autoimmune disorder varies from person to person and from place to place depending upon the immune status of the patient.

Aims \& objectives:

To describe the fluorescence pattern of ANA by indirect immunofluorescent assay in patients with autoimmune diseases and compare it with their ANA profile.

\section{Material \& Methods:}

In this retrospective study (January 2018 to December 2020) we reviewed the case records of 96 patients with different autoimmune diseases. Their demographic details like age, gender, family history, presenting symptoms were tabulated. We compared their respective ANA test reports (Pattern and profile)

Results: Data 96 patients (20 male and 76 females) was analyzed. The age range of the study cohort was 10- 77 years, mean age was 39.53. There were 23(23.9\%) patients with SLE, 34(35.4\%) with MCTD. 6 (6.2\%) Sjogren's syndrome. Glomerulonephritis was present in 7(7.2\%) patients and Rheumatoid arthritis in $4(4.1 \%)$. All study participants had ANA positive and ANA pattern was seen as homogenous in $34(35.4 \%)$, speckled $45(46 \%)$, nuclear pattern was seen in 33(34.9\%). LIA was found to be significantly positive in sjogren's disease while in SLE only 22 patients had positive LIA. None of our Rheumatoid arthritis patients had positive LIA

Conclusion: We conclude that it is better to restrict performing line immunoassays as these are expensive and use ANA-IIF fluorescent patterns to predict presence of autoantibodies to diagnose an autoimmune disease.

\section{Introduction:}

Connective tissue diseases (CTD) are a group of autoimmune systemic diseases that can affect any organ-system in the body. The initial clinical presentations of these diseases overlap, not only with each other, but also with a wide range of other nonrheumatological disorders. Due to these reasons, clinicians depend on the use of the immunology laboratory for clinching the diagnosis. A large number of tests exist in the laboratory for the investigation of CTD and each test can be performed by a number of different methods, albeit with its own limitations.
ANAs represent key biomarkers in the evaluation of autoimmune diseases. The fluorescent ANA assay is often viewed to be the gold standard. The presence of antinuclear antibodies (ANAs), which include auto antibodies to extractable nuclear antigens (ENAs), in the sera of patients with autoimmune diseases provides useful immunologic and pathophysiologic insights into the nature of their disease in addition to diagnosis.(1)The comprehensive description of 29 different ANA-IIF patterns by the international consensus of ANA patterns (ICAP) facilitates the 
harmonization of ANA-IIF diagnostics. These diverse groups of autoantibodies recognize nuclear macromolecules and their complexes. Nevertheless, ANAs might be expressed in autoimmune diseases like SLE less commonly than often thought.(2)

The other side of the coin is that the positive ANA tests are frequently observed in healthy individuals and a reason for referral to rheumatologists. In these cases, additional tests are required, for example, the detection of anti-DFS70 antibodies can be helpful to exclude systemic autoimmune rheumatic diseases.(3)

Commercially there are a lot of tests that evaluate ANA. However, Tan et al concluded that no single manufacturer was clearly superior to others in terms of their products' overall sensitivity, specificity, and precision.(4) Furthermore, Sebastian et al in their study concluded that the screening of sera by ANA-IIF method alone may suffice and probably reduce the expense of detailed immunological work-up with minimal loss in diagnostic accuracy. (5)

It is well known that the immunity status, individual response to disease and types of antibodies produced vary from person to person and probably from population to population. Thus the distribution and autoantibody profiles need to be studied in a particular catchment area of a given institution. Hence we were prompted to undertake this study, wherein we analysed a cohort of 96 patients with autoimmune diseases and compared their ANA pattern \& profile .

\section{Materials and Methods:}

In this retrospective study, we retrieved and reviewed the case records of patients with different autoimmune diseases. We tabulated their demographic details like age, gender, family history and compared their respective ANA test reports (Pattern and profile). The study cohort of patients had presented with symptoms varying from joint pains, fever and fatigue etc classical of an autoimmune disease and were evaluated at Yenepoya Medical college Mangalore, a tertiary care center in the southern Indian state of Karnataka between January 2018 to December 2020. The study was approved by the scientific review board and there was clearance from the institutional ethics committee.

The immunological investigation data were obtained from the archives of the Department of Microbiology of the hospital.
At our centre, ANA in serum is detected using the EUROIMMUN titer-plane technique. The kit comprises Biochip slides embedded with HEp-2010/liver (monkey) tissue sections. As per the manufacturer protocol, serum samples diluted 1:100 with phosphate buffer saline (PBS)-Tween followed by tagging with fluorescein-labelled anti human globulin. LIA is an indirect membrane based enzyme immunoassay for the qualitative measurement of Ig G class antibodies against dsDNA, nucleosome, histone, SmD1, PCNA, ribosomal, P0(RPP,SS-A/Ro-60,SSA/Ro-52,SS-B/La, CENP-B, Sc170,U1-snRNP , AMA M2 ,Jo-1,PM-Scl,Mi-2\& Ku in human serum or plasma . The test is based on the principle of line immuno assay (LIA). Nuclear and associated cytosolic antigens are applied as lines on a nitrocellulose membrane. Nitrocellulose membrane is blocked to prevent nonspecific reactions. During incubation of a strip with diluted patient samples autoantibodies present in the sample bind to the antigen on the strip.

\section{Results}

There were 96 (20 male and 76 females) patients with autoimmune diseases during the study period. The age range of the study cohort was 10- 77 years, mean age was 39.53. Clinical manifestations suggestive of autoimmune diseases were Fever, fatigue, arthritis, renal manifestations and oral ulcers. The detailed symptom pattern in ANA positive patients is shown in Table 1.

Majority of our study cohort had SLE followed by, MCTD, sjogren's syndrome, rheumatoid arthritis and dermatomyositis. The details are shown in Table 2

ANA pattern was positive in all samples while LIA was positive in 40 samples $(41.6 \%)$

The homogenous pattern was the most common ANA pattern, seen in 32( $33.33 \%$ ) patients .

The second most commonly occurring ANA pattern in this series was the speckled $(\mathrm{n}=15 ; 15.62 \%)$ pattern. Nucleolar pattern was observed in none of the patients in this study. Details can be seen in the table 3

Line immunoassay showed positivity for U1-snRNP in $13(14.5 \%)$, followed by SSA/Ro60 $11(11.45 \%)$ SSA/Ro 52, anti dsDNA5(5.20\%) histone 3(3.12\%), nucleosome 3(3.12\%)AMA M2 2(2.08\%),Mi 22 (2.08\%), Details are shown in Table No 4

Strangely, $56(58.33 \%)$ of the ANA-IIF positive samples showed negativity with line immunoassay. 


\begin{tabular}{|c|c|}
\hline & Table 1: Demographic details $N=96$ \\
\hline Gender distribution & 26 Males, 70 females \\
\hline Age range: & $10-76$ years \\
\hline Mean age: & 39.53 years \\
\hline Symptoms & Fever $31(32.2 \%)$ \\
\hline & Fatigue $16(16.6 \%)$ \\
\hline & Arthritis $5(7.2 \%)$ \\
\hline & Oral ulcers $3(3.1 \%)$ \\
\hline & Renal manifestations $15(15.6 \%)$ \\
\hline & $\operatorname{Rash} 7(7.2 \%)$ \\
\hline & Morning stiffness $4(4,1 \%)$ \\
\hline & Skin lesions $10(10.4 \%)$ \\
\hline & Alopecia 6(6.2\%) \\
\hline & Photosensitivity $10(10.4 \%)$ \\
\hline & Joint pains $20(20.8 \%)$ \\
\hline & GIT symptoms $20(20.8 \%)$ \\
\hline
\end{tabular}

Table 2: Diagnostic profile of the patients N-96

\begin{tabular}{|l|l|}
\hline SLE & $23(23.9 \%)$ \\
\hline MCTD & $16(16.6 \%)$ \\
\hline Glomerulonephritis & $7(7.29 \%)$ \\
\hline $\begin{array}{l}\text { Sjogren's Syndrome } \\
\text { Limited Scleroderma }\end{array}$ & $6(6.25 \%)$ \\
\hline Rheumatoid arthritis & $4(4.1 \%)$ \\
\hline Dermatomyositis & $3(3.1 \%)$ \\
\hline Oral Submucosal fibrosis & $2(2 \%)$ \\
\hline Cutaneous small vessel Vasculitis & $3(3 \%)$ \\
\hline Reactive arthirtis & $3(3 \%)$ \\
\hline Miscellaneous & $29(30 \%)$ \\
\hline
\end{tabular}


Table 3: ANA pattern $\mathrm{N}=96$

\begin{tabular}{|l|l|}
\hline Homogenous & $32(33 \%)$ \\
\hline Speckled & $15(15.6 \%)$ \\
\hline Granular & $7(7.2 \%)$ \\
\hline Mixed & $3(3.1 \%)$ \\
\hline Rim & $2(2 \%)$ \\
\hline
\end{tabular}

Table 4: LIA pattern $\mathrm{N}=41$

\begin{tabular}{|l|l|}
\hline U1-snRNP & $13(31.7 \%)$ \\
\hline SSA/Ro60 & $11(26.8 \%)$ \\
\hline SSA/R052 & $8(19.5 \%)$ \\
\hline dSDNA & $5(12 \%)$ \\
\hline SmD1 & $5(12 \%)$ \\
\hline Nucleosomes & $3(7.3 \%)$ \\
\hline Histones & $3(7.3 \%)$ \\
\hline AMA M2 positive & $2(4.8 \%)$ \\
\hline Mi-2 & $2(4.8 \%)$ \\
\hline CENP-B, Strong positive & $1(2.4 \%)$ \\
\hline
\end{tabular}

\section{Discussion:}

The presence of ANA is a hallmark of autoimmune disease and is widely used to diagnose various autoimmune disorders. The results of our study showed that autoimmune disorders were more common among females and SLE was a major autoimmune illness followed by Mixed connective disease as shown in Table 2 which is in line with other studies $(2,3,6)$

While all our Rheumatoid arthritis patients had positive ANA none of them had positive LIA. Contrary to this, all patients with Sjogren's disease had positive ANA levels and $5 / 6(83 \%)$ had LIA positive (SSA/Ro60, SSA/Ro52, SSB/La).

LIA positivity has been linked to prognosis of Sjogren's disease $(\mathrm{SjS})$ and hence it becomes mandatory to test for LIA in a given patient in addition to ANA testing. Brito-Zerón et al observed a strong influence of immunological markers on the phenotype of primary $\mathrm{SjS}$ at diagnosis in the largest multi-ethnic international cohort. The authors in this study concluded that the immunological patterns may play a central role in the phenotypic expression of the disease at the time of diagnosis, and thus guide physicians to design a specific personalised management during the follow-up of patients with primary SjS.(7) .

A hierarchical testing system for the investigation of CTD when used appropriately, in conjunction with the clinical picture, can result in the diagnosis/exclusion of CTD more efficiently and economically. In contrast, random use of the laboratory tests, combined with limited knowledge of the methods used to carry out these tests, can lead to delay or even misdiagnosis, as well as can lead to wastage of resources. (8)

While observing the association between immunofluorescence patterns of antinuclear antibody on HEp-2 cell and more specific antinuclear reactivities (e.g. anti-dsDNA and anti-extractable nuclear antigen) 
in the serum samples of connective tissue disorders (CTD) Sharmeen et al observed that of 152 CTD patients $110(72.3 \%)$ cases were ANA positive by IIF on HEp-2 cell in their study cohort. All our patients with SLE had ANA positivity. In our study majority of ANA had a homogenous pattern as shown in table 3 . Our results are partially in line with these workers (9)

In our study there were 23 patients with SLE and ds DNA was positive in $4(27.3 \%)$ patients with SLE. Two patients with lupus nephritis had positive SmD1 while 3 patients had anti histone positivity. In a cohort of 1390 patients it was observed by Sulcebe et al that the Positive predictive value were found to be appreciable only for systemic lupus erythematosus (SLE); the anti-ENA positive and anti-nDNA positive (10) The SLE patients generally showed higher frequencies of renal disease, as was observed in this study.

We observed a high sensitivity of ANA in diagnosing SLE. In a cohort of 1010 samples Slater et al observed that the sensitivity of the ANA test for SLE was high. However, the authors concluded that the clinicians ordering ANA test should be aware of the test's lowpositive predictive value in settings with a low prevalence of rheumatic disease, particularly among older patients.(11)

Two patients in our study had undifferentiated connective tissue disease and had positive ANA and their ANA profile was AMA M2 positive. Both patients had presented with fever and fatigue with a high clinical suspicion of autoimmunity.

While ANA was positive in all 17 patients with MCTD only 11 patients had positive LIA as shown in table 3 in this study. Anti-U1-RNP positivity remains mandatory for the mixed connective tissue disease (MCTD) diagnosis. In our study anti-U1-RNP was positive in 5 out of 11 MCTD (45\%). In a previous study, the prevalence of $25-30 \%$ for anti-RNP was reported in John Hopkins and LUMINA lupus cohorts and also $13 \%$ prevalence for the anti-U1-RNP in EuroLupus cohort. Presence of anti-U1-RNP antibodies in patients fulfilling SLE criteria (but not the MCTD ones) was associated with manifestations such as Raynaud phenomenon, musculoskeletal and lung impairment some clinical features frequently encountered in MCTD patients and only rarely described in lupus population. The use of more specific markers such as $70 \mathrm{kDa}$ anti-U1-RNP or anti-Sm-D was proposed for discriminating between SLE and MCTD by the authors (12) The occurrence of evolution from MCTD to another defined rheumatic condition, and the prevalence and durability of remission after long-term observation was studied by Resister $\mathrm{S}$ et al. Their results strengthen the view of MCTD as a relatively stable disease entity. Long-term remission in MCTD is not frequent; however, the low SLEDAI-2 K and EUSTAR scores during the observation period suggests that the disease runs a milder course than systemic lupus erythematosus and systemic sclerosis. (13) Thus these antibodies help in prognosis of the disease in the long run.

To conclude the results of this study provide a reference database for the local population in Southern India. We conclude that it is better to restrict performing line immunoassays in all patients with autoimmunity, as these are expensive and use ANA-IIF fluorescent patterns to predict presence of autoantibodies to precisely diagnose the autoimmune disease.

Financial disclosure: There were no financial implications

Ethical approval: Study was approved by the ethical committee of the institution.

\section{Reference:}

1. Ling M, Murali M. Antinuclear Antibody Tests. Clin Lab Med. 2019 Dec;39(4):513-24.

2. Pisetsky DS. Antinuclear antibody testing misunderstood or misbegotten? Nat Rev Rheumatol. 2017 Aug;13(8):495-502.

3. Rose T, Dörner T. [Antinuclear antibodies in the diagnostics of rheumatic diseases]. Z Rheumatol. 2020 Jun;79(5):447-58.

4. Tan EM, Smolen JS, McDougal JS, et al. A critical evaluation of enzyme immunoassays for detection of antinuclear autoantibodies of defined specificities. I. Precision, sensitivity, and specificity. Arthritis Rheum. 1999 Mar;42 (3): 455-64.

5. Sebastian W, Roy A, Kini U, et al Correlation of antinuclear antibody immunofluorescence patterns with immune profile using line immunoassay in the Indian scenario. Indian J Pathol Microbiol. 2010 Sep;53(3):427-32.

6. Berner ES, Webster GD, Shugerman AA, et al. Performance of four computer-based diagnostic systems. N Engl J Med. 1994 Jun 23;330(25):1792-6.

7. Brito-Zerón P, Acar-Denizli N, Ng WF, et al. How immunological profile drives clinical phenotype of primary Sjögren's syndrome at diagnosis: analysis of 10,500 patients (Sjögren Big Data Project). Clin 
Exp Rheumatol. 2018 May-Jun;36 Suppl 112(3):102-112.

8. Aziz KA, Faizal AA. The role of the clinical immunology laboratory in the diagnosis and monitoring of connective tissue diseases. Saudi Med J. 2004 Dec;25(12):1796-807.

9. Sharmin S, Ahmed S, Abu Saleh A, et al. Association of Immunofluorescence pattern of Antinuclear Antibody with Specific Autoantibodies in the Bangladeshi Population. Bangladesh Med Res Counc Bull. 2014 Aug;40(2):74-8.

10. Sulcebe G, Morcka K. Diagnostic and prognostic significance of different antinuclear antibodies in more than 1000 consecutive Albanian patients with rheumatic diseases. Clin Exp Rheumatol. 1992 May-Jun;10(3):255-61. PMID: 1582070.

11. Slater CA, Davis RB, Shmerling RH. Antinuclear antibody testing. A study of clinical utility. Arch Intern Med. 1996 Jul 8;156(13):1421-5.

12. Dima A, Jurcut C, Baicus C. The impact of antiU1-RNP positivity: systemic lupus erythematosus versus mixed connective tissue disease. Rheumatol Int. 2018 Jul;38(7):1169-78.

13. Reiseter S, Gunnarsson R, Corander J, et al. Disease evolution in mixed connective tissue disease: results from a long-term nationwide prospective cohort study. Arthritis Res Ther. 2017 Dec 21;19(1):284. 